\title{
On the Homotopy Type of the Clique Graph*
}

\author{
F. Larrión ${ }^{a, 1}$ V. Neumann-Lara ${ }^{a, 1}$ M. A. Pizaña ${ }^{b}$ \\ ${ }^{a}$ Instituto de Matemáticas, U.N.A.M. \\ Circuito Exterior, C.U. México 04510 D.F. MÉXICO. \\ \{paco, neumann\}@matem.unam.mx
${ }^{b}$ Universidad Autónoma Metropolitana, Depto. de Ingeniería Eléctrica. Av. Michoacán y Purísima s/n México 09340 D.F. MÉXICO. map@xanum.uam.mx, http://xamanek.uam.mx/map

\begin{abstract}
If $G$ is a graph, its clique graph $K(G)$ is the intersection graph of all its (maximal) cliques. The complex $G^{\uparrow}$ of a graph $G$ is the simplicial complex whose simplexes are the vertex sets of the complete subgraphs of $G$.

Here we study a sufficient condition for $G^{\uparrow}$ and $K(G)^{\uparrow}$ to be homotopic. Applying this result to Whitney triangulations of surfaces, we construct an infinite family of examples which solve in the affirmative Prisner's open problem 1 in Graph Dynamics (Longman, Harlow, 1995): Are there finite connected graphs $G$ that are periodic under $K$ and where the second modulo 2 Betti number is greater than 0 ?

Keywords: clique graphs, clique convergence, Whitney triangulations, clean triangulations, simplicial complexes, modulo 2 Betti numbers.
\end{abstract}

\section{Introduction and terminology}

All our graphs are simple. If $G$ is a graph, a complete of $G$ is a complete subgraph of $G$ and a clique is a maximal complete of $G$. The clique number $\omega(G)$ is the maximum order of a clique of $G$. We shall often identify induced subgraphs with their vertex sets. In particular, we shall often write $x \in G$ instead of $x \in V(G)$.

We say that $G$ is locally $H$ if the subgraph $N_{G}(x)$ induced in $G$ by the (open) neighbourhood of any vertex $x \in G$ is isomorphic to $H$. We say

\footnotetext{
${ }^{*}$ Dedicated to Prof. J. L. Szwarcfiter in his $60^{t h}$ Anniversary.

${ }^{1}$ Partially supported by CONACyT, Grant 400333-5$27968 \mathrm{E}$.
}

that $G$ is locally $\mathcal{H}=\left\{H_{1}, H_{2}, \ldots\right\}$ if for every $x \in G, N_{G}(x) \cong H_{i}$ for some $H_{i} \in \mathcal{H} . C_{n}$ and $P_{n}$ are, respectively, the cyclic and path graphs on $n$ vertices. We say that $G$ is locally cyclic if it is locally $\left\{C_{n}: n \geq 3\right\}$.

The clique graph $K(G)$ of $G$ has all cliques of $G$ as vertices, two of them being adjacent iff they (are different and) share some vertex of $G$. We call $K$ the clique operator. Iterated clique graphs are inductively defined by $K^{0}(G)=G$ and $K^{n+1}(G)=K\left(K^{n}(G)\right)$. $G$ is $K$-periodic if $G \cong K^{n}(G)$ for some $n \geq 1$. Extensive bibliography on clique graphs can be found in [14].

A graph $G$ is clique-Helly if whenever $X=$ $\left\{q_{1}, \ldots, q_{n}\right\} \subseteq V(K(G))$ is a family of pairwise intersecting cliques, then $\bigcap X \neq \varnothing$. We say that $Q=\left\{q_{1}, \ldots, q_{n}\right\} \in V\left(K^{2}(G)\right)$ is a star of $G$ if $\bigcap Q \neq \varnothing$, otherwise it is a necktie of $G$. Obviously, a graph is clique-Helly iff it has no necktie.

If $G$ is a graph, $G^{\uparrow}$ is the simplicial complex whose simplexes are the completes of $G$. We say that two simplicial complexes are homotopic $(\mathbb{K} \simeq \mathbb{L})$ when their geometric realizations are homotopic $(|\mathbb{K}| \simeq|\mathbb{L}|)$. The behaviour of topological invariants of $G^{\uparrow}$ under several graph operators (including the clique operator) has been studied in $[9,10,11]$. In particular, Prisner proved in $[10]$ that if $G$ is clique-Helly, $G^{\uparrow} \simeq K(G)^{\uparrow}$. Our main result (Theorem 2.4) states that this is also true for many non-clique-Helly graphs. As an application of this, we will show (Theorem 2.5) that if $G$ is free of tetrahedra and induced octahedra, then $G^{\uparrow} \simeq K(G)^{\uparrow}$.

An interesting particular case is when the realization $|\mathbb{K}|$ is a compact surface (with or without border), i.e. $\mathbb{K}$ is a triangulation of a compact surface. If $G$ is the underlying graph (or 1- 
skeleton) of a surface triangulation $\mathbb{K}$, every face of $\mathbb{K}$ is a triangle of $G$ but the converse may not be true. We shall be interested in surface triangulations where every triangle of $G$ is a face of $\mathbb{K}$ : such a triangulation is a Whitney triangulation [17]. Thus, if $\mathbb{K}$ is Whitney it is determined by $G$, and we tend to identify $G$ with $\mathbb{K}$, and sometimes even with $|\mathbb{K}|$. If $\mathbb{K}$ is Whitney, (except for the tetrahedron $K_{4}$ ) the cliques of $G$ are precisely the faces of the triangulation. Whitney triangulations have other names and have been studied before $[2,5,8,16,17]$. In particular, the description of the dynamical behaviour under the clique operator of the regular Whitney triangulations has been completed in [8]. As a corollary to our Theorem 2.5 , we will have that the only Whitney triangulation of a compact surface which is not homotopic to its clique graph is the octahedron. We shall use the following two theorems:

Theorem 1.1 [8] $G$ is the underlying graph of a Whitney triangulation of a closed surface (resp. compact surface) if and only if $G$ is locally cyclic (resp. $G$ is locally $\left\{C_{n}, P_{m}: n \geq 3, m \geq 2\right\}$ ).

Theorem 1.2 [8] For every Whitney triangulation $G$ of a closed surface with minimum degree at least 7 we have $K(G) \cong K^{3}(G)$.

We refer to [1], [10] and [13] for undefined concepts.

\section{Homotopy}

If $\mathcal{H}$ is a hypergraph, $\mathcal{H}^{*}$ denotes its dual hypergraph, and $\mathcal{H}^{\downarrow}$ is the smallest simplicial complex containing the hyperedges of $\mathcal{H}$ as simplexes. The following reformulation is due to Prisner $[9,10]$ :

Theorem 2.1 (Dowker, [3]) For every hypergraph $\mathcal{H}, \mathcal{H}^{\downarrow}$ and $\mathcal{H}^{* \downarrow}$ are homotopic.

If $G$ is a graph, $\mathcal{K}(G)$ is its clique hypergraph: $\mathcal{K}(G)$ has the same vertex set as $G$ and its hyperedges are the cliques of $G$. It follows immediately from the definitions that $G^{\uparrow}=\mathcal{K}(G)^{\downarrow}$.

The star hypergraph $\mathcal{S}(G)$ of $G$ has the same vertex set as $K(G)$ and the hyperedges are the cliques $Q=\left\{q_{1}, q_{2}, \ldots, q_{r}\right\}$ of $K(G)$ satisfying $\bigcap Q \neq \varnothing$. It follows that $\mathcal{K}(G)^{* \downarrow}=\mathcal{S}(G)^{\downarrow}$ and that $\mathcal{S}(G)^{\downarrow} \subseteq K(G)^{\uparrow}$. The equality $\mathcal{S}(G)^{\downarrow}=$ $K(G)^{\uparrow}$ holds precisely when $G$ is clique-Helly.
Then, as pointed out by Prisner [10, Proposition $2.2]$, it follows from Dowker's theorem that $G^{\uparrow}$ and $K(G)^{\uparrow}$ are homotopic for every clique-Helly graph $G$. A reformulation of this result will be useful to us:

Theorem 2.2 (Prisner, [10]) For every graph $G$, we have $G^{\uparrow}=\mathcal{K}(G)^{\downarrow} \simeq \mathcal{K}(G)^{* \downarrow}=\mathcal{S}(G)^{\downarrow} \subseteq$ $K(G)^{\uparrow}$. In particular, if $G$ is clique-Helly, then $G^{\uparrow} \simeq K(G)^{\uparrow}$.

Prisner provided examples of graphs $G$ (namely the $n$-dimensional octahedra, for $n \geq 3$ ) such that $G^{\uparrow}$ and $K(G)^{\uparrow}$ are not homotopic. As we shall see shortly, this property of the octahedra is tightly connected to the fact that octahedra contain neckties without a center.

Definition 2.3 If $X$ is a complete of $K(G)$ satisfying $\bigcap X=\varnothing$, then $q_{0} \in K(G)$ is called a center of $X$ if:

$Y \subseteq X$ and $\bigcap Y \neq \varnothing$ imply $\bigcap\left(Y \cup\left\{q_{0}\right\}\right) \neq \varnothing$.

Note that $X \cup\left\{q_{0}\right\}$ is always a complete of $K(G)$. Also, when such an $X$ is a clique of $K(G)$, $X$ must contain all its centers.
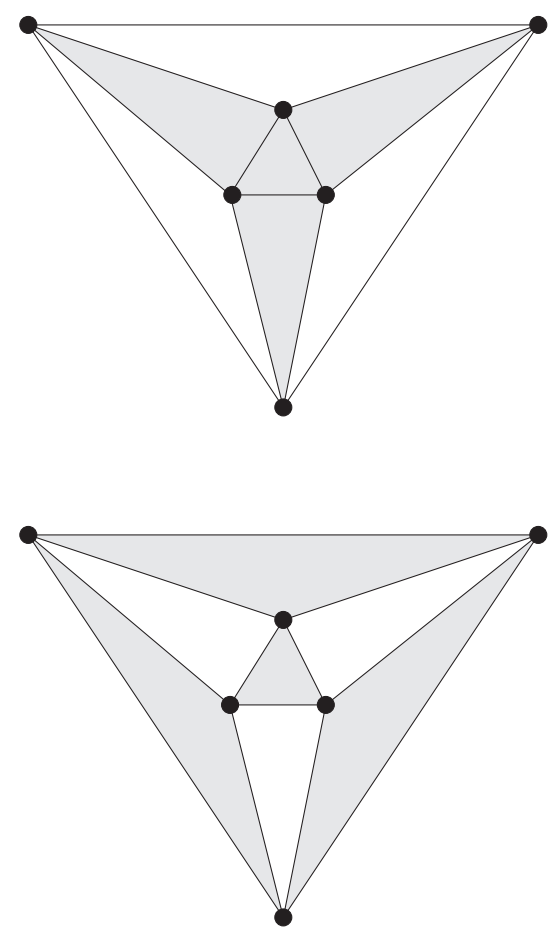

Figure 1: Two neckties of the octahedron, with center (above) and without center (below). 
Many non-Helly graphs $G$ satisfy $G^{\uparrow} \simeq K(G)^{\uparrow}$, Indeed we shall show that for many non-Helly graphs $G, \mathcal{S}(G)^{\downarrow}$ is a strong deformation retract of $K(G)^{\uparrow}$.

Let's rename $\mathbb{S}=\mathcal{S}(G)^{\downarrow}$ and $\mathbb{K}=K(G)^{\uparrow}$. We know that $\mathbb{S} \subseteq \mathbb{K}$. Note that the 0 -simplexes of $\mathbb{S}$ and $\mathbb{K}$ are the same. In order to easily define the required mappings, we take the barycentric subdivision $\mathbb{K}^{\prime}$ of $\mathbb{K}$ relative to $\mathbb{S}$ as used in $[12$, page 19].

Equivalently, we define the complex $\mathbb{K}^{\prime}$ whose vertices are those of $\mathbb{K}$ (denoted by $q_{i}$ ) plus a (formal) barycenter $b(s)$ for each $s \in$ $\mathbb{K}-\mathbb{S}$, and whose simplexes are of the form $\left\{q_{1}, \ldots, q_{n}, b\left(s_{1}\right), \ldots, b\left(s_{m}\right)\right\}$ and satisfy:

$$
\begin{aligned}
& \text { 1. }\left\{q_{1}, \ldots, q_{n}\right\} \in \mathbb{S} . \\
& \text { 2. } s_{j} \in \mathbb{K}-\mathbb{S} \text { for all } j . \\
& \text { 3. } q_{i} \in s_{1} \text { for all } i . \\
& \text { 4. } s_{j} \subseteq s_{j+1} \text { for all } j .
\end{aligned}
$$

and then we may prove that this is indeed a subdivision of $\mathbb{K}$ using Theorem 3.3.4 in [13]. Of course, we still have $\mathbb{S} \subseteq \mathbb{K}^{\prime}$.

The idea behind this is to grab the offending simplexes (those in $\mathbb{K}-\mathbb{S}$ ) by its barycenters and retract them into $\mathbb{S}$. Now we can prove our main result:

Theorem 2.4 Let $G$ be a graph. Assume that any complete $X$ of $K(G)$ with $\bigcap X=\varnothing$ has a center which belongs to every necktie containing $X$. Then $\mathcal{S}(G)^{\downarrow}$ is a strong deformation retract of $K(G)^{\uparrow}$. In particular, $G^{\uparrow} \simeq K(G)^{\uparrow}$.

Proof. For every simplex $s$ in $\mathbb{K}-\mathbb{S}$ select, using the hypothesis, a fixed center $q(s)$ of $s$ belonging to every maximal simplex (i.e. necktie) that contains $s$. Also, for each $s \in \mathbb{K}-\mathbb{S}$, define

$$
\widehat{s}=\bigcap\left\{Q \in K^{2}(G): s \subseteq Q\right\} .
$$

Note: $s \subseteq \widehat{s} \in \mathbb{K}-\mathbb{S}$, and $s \subseteq s^{\prime}$ implies $q(\widehat{s}) \in$ $\widehat{s} \subseteq \widehat{s^{\prime}}$.

Now define the map $\varphi_{1}: \mathbb{K}^{\prime} \rightarrow \mathbb{K}$ by $\varphi_{1}\left(q_{i}\right)=q_{i}$ and $\varphi_{1}\left(b\left(s_{j}\right)\right)=q\left(\widehat{s_{j}}\right)$. Then for any simplex of $\mathbb{K}^{\prime}$ we have that $\varphi_{1}\left(\left\{q_{1}, \ldots, q_{n}, b\left(s_{1}\right), \ldots, b\left(s_{m}\right)\right\}\right)=$ $\left\{q_{1}, \ldots, q_{n}, q\left(\widehat{s_{1}}\right), \ldots, q\left(\widehat{s_{m}}\right)\right\}$. This is a simplex of $\mathbb{K}$ because there is a clique $Q$ of $K(G)$ such that $q_{i} \in Q$ and $s_{j} \subseteq Q$ for all $i=1, \ldots, n$ and $j=1, \ldots, m$ (take a $Q$ with $s_{m} \subseteq Q$ ). Therefore $q_{i}, q\left(\widehat{s_{j}}\right) \in Q$ for all $i$ and $j$. It follows that $\varphi_{1}: \mathbb{K}^{\prime} \rightarrow \mathbb{K}$ is a simplicial map, so $\left|\varphi_{1}\right|:\left|\mathbb{K}^{\prime}\right| \rightarrow|\mathbb{K}|$ is continuous.
We claim now that $\operatorname{Im}\left(\varphi_{1}\right)=\mathbb{S}$ : As $q_{1} \cap \cdots \cap$ $q_{n} \neq \varnothing$ and $\left\{q_{1}, \cdots, q_{n}\right\} \subseteq s_{1} \subseteq \widehat{s_{1}}$, we obtain that $q_{1} \cap \cdots \cap q_{n} \cap q\left(\widehat{s_{1}}\right) \neq \varnothing$. Using that $\widehat{s_{1}} \subseteq$ $\widehat{s_{2}} \subseteq \cdots \subseteq \widehat{s_{m}}$ and $q\left(\widehat{s_{j}}\right) \in \widehat{s_{j}}$ for all $j$, it follows by induction that $\left\{q_{1}, \ldots, q_{n}, q\left(\widehat{s_{1}}\right), \ldots, q\left(\widehat{s_{m}}\right)\right\}$ is a simplex of $\mathbb{S}$. Now we know that $\operatorname{Im}\left(\left|\varphi_{1}\right|\right)=|\mathbb{S}|$ and that the restriction of $\left|\varphi_{1}\right|$ to $|\mathbb{S}|$ is the identity in $|\mathbb{S}|$.

On the other hand, consider the canonical homeomorphism $\varphi_{0}:|\mathbb{K}| \rightarrow\left|\mathbb{K}^{\prime}\right|$. Let $\varphi=$ $\left|\varphi_{1}\right| \circ \varphi_{0}$. Note that for all $x \in|\mathbb{K}|$ there is a simplex $s \in \mathbb{K}$ such that $x, \varphi(x) \in|s|$ (any maximal simplex $s \in \mathbb{K}$ satisfying $x \in|s|$ will do). Then it follows that $\varphi \simeq 1_{|\mathbb{K}|}$ via the homotopy $H(x, t)=t x+(1-t) \varphi(x)$ (see, for example $[6$, Prop. 1.7.5]). Since $\left.\varphi\right|_{|\mathbb{S}|}=1_{|\mathbb{S}|}$, we have that $H(x, t)=x$ for all $x \in|\mathbb{S}|$. Therefore $|\mathbb{S}|$ is a strong deformation retract of $|\mathbb{K}|$.

An interesting consequence is the following:

Theorem 2.5 If $G$ is a graph free of induced octahedra and $\omega(G) \leq 3$, then $G^{\uparrow} \simeq K(G)^{\uparrow}$

Proof. Without loss of generality we assume $G$ to be connected and non-trivial. Then we observe that every clique of $G$ is a triangle or an edge.

Let $X$ be a complete of $K(G)$ satisfying $\bigcap X=$ $\varnothing$, and let $Z=\left\{q_{1}, \ldots, q_{r}\right\}$ be a minimal subset of $X$ also satisfying $\cap Z=\varnothing$.

Since $Z$ is minimal and necessarily $r \geq 3$, we may take $x_{23} \in \bigcap\left(Z-\left\{q_{1}\right\}\right), x_{13} \in \bigcap(Z-$ $\left.\left\{q_{2}\right\}\right)$ and $x_{12} \in \bigcap\left(Z-\left\{q_{3}\right\}\right)$. Hence, $q_{0}=$ $\left\{x_{12}, x_{13}, x_{23}\right\}$ is a clique of $G$. This very construction was used by J. L. Szwarcfiter in his celebrated characterization of clique-Helly graphs [15].

It follows that $q_{1}=\left\{x_{12}, x_{13}, a\right\}, q_{2}=$ $\left\{x_{12}, x_{23}, b\right\}$ and $q_{3}=\left\{x_{13}, x_{23}, c\right\}$ for some three (different) vertices $a, b, c \in G$. Since $q_{1} \cap q_{2} \cap q_{3}=$ $\varnothing$ it follows that $Z=\left\{q_{1}, q_{2}, q_{3}\right\}$.

Let $Q \in K^{2}(G)$ be a necktie containing $Z$, and let $q \in Q$. If $q \cap q_{0}=\varnothing$, then $q=\{a, b, c\}$ and the set of vertices $\left\{x_{12}, x_{13}, x_{23}, a, b, c\right\}$ induces an octahedron in $G$, contradicting our hypotheses. If $\left|q \cap q_{0}\right|=1$, say $q \cap q_{0}=\left\{x_{12}\right\}$, then $q \cap q_{3}=\{c\}$ and $\left\{x_{12}, x_{13}, x_{23}, c\right\}$ would contradict $\omega(G) \leq 3$. Therefore $\left|q \cap q_{0}\right| \geq 2$ for every $q \in Q$.

Since the set $\left\{q \in K(G):\left|q \cap q_{0}\right| \geq 2\right\}$ is a complete of $K(G)$ it follows that $Q=\{q \in K(G)$ : $\left.\left|q \cap q_{0}\right| \geq 2\right\}$. Now the condition on the clique number implies that $q_{0}$ is a center of $Q$. Then $Q$ is the unique necktie containing $Z$, so it is also unique containing $X$. Therefore $q_{0}$ is a center of $X$ which belongs to every necktie containing $X$, and we apply the previous theorem. 
The following result is an immediate consequence:

Corollary 2.6 The only Whitney triangulation of a compact surface (with or without border) which is not homotopic to its clique graph is the octahedron.

Now let's denote the $i$-th modulo 2 Betti number of a complex $\mathbb{K}$ by $\hat{\beta}_{i}(\mathbb{K})$. Take any locally $\left\{C_{t}: t \geq 7\right\}$ graph $H$. By Theorem 1.1 $H$ is a Whitney triangulation of a closed surface, so we have $\hat{\beta}_{2}\left(H^{\uparrow}\right)=1$. Since $H^{\uparrow} \simeq K(H)^{\uparrow}$, we have $\hat{\beta}_{2}\left(K(H)^{\uparrow}\right)=1$. Then Theorem 1.2 tells us that $G:=K(H)$ is $K$-periodic, thus solving Prisner's open problem 1 in [11].

As a concrete example, it is shown in [8] that $I \times$ $K_{3}$ is a locally $C_{10}$ graph (here $I$ is the icosahedron and $\left\{(a, b),\left(a^{\prime}, b^{\prime}\right)\right\} \in E(A \times B)$ iff $\left\{a, a^{\prime}\right\} \in E(A)$ and $\left.\left\{b, b^{\prime}\right\} \in E(B)\right)$. In fact, Brown and Connelly [2] proved that for every $t$ there is at least one finite locally $C_{t}$ graph. Next, we shall construct an explicit infinite family of locally $C_{7}$ graphs.

\section{Whitney triangulations}

Let's start with an infinite graph $T: V(T)=$ $\mathbb{Z} \oplus \mathbb{Z}$ and put $N=\{ \pm(1,0), \pm(0,1), \pm(1,-1)\}$, then define $\{x, y\} \in E(T)$ if and only if $y-x \in N$.

Each vector $u \in \mathbb{Z} \oplus \mathbb{Z}$ gives rise to a translation $x \mapsto u+x$ which is an automorphism of $T$. Every finite locally $C_{6}$ graph triangulating the torus is a quotient $T / \Gamma$ where $\Gamma$ is the translation group generated by the translations given by two linearly independent vectors $u, v \in \mathbb{Z} \oplus \mathbb{Z}$. The group $\Gamma$ must satisfy the following admissibility condition: for every $\gamma \in \Gamma$ and $v \in V(T)$, the distance in $T$ from $v$ to $\gamma(v)$ is at least 4 (otherwise, the resulting triangulation is not Whitney, see [7]).

Let $u=(4,1)$, fix $r \geq 2$, and let $v_{r}=(2 r, 4 r)$. Let $\Gamma_{r}$ be the translation group defined by $u$ and $v_{r}$, and let $\mathcal{P}_{r}$ be the parallelogram defined by these two vectors. The locally $C_{6}$ graph $G_{r}=$ $T / \Gamma_{r}$ defines a Whitney triangulation of the torus with $14 r$ vertices: $G_{r}$ is obtained by identifying the parallel edges of $\mathcal{P}_{r}$.

Now consider the $2 r$ vertices $w_{1}, w_{2}, \ldots, w_{2 r}$ of $G_{r}$ which correspond to the vertices $(2,1),(3,3), \ldots,(2 r+1,4 r-1)$ in $\mathcal{P}_{r}$, i.e. $w_{i}=(i+1,2 i-1)$. The vertices of $G_{r}$ are the disjoint union of the closed neighbourhoods $N\left[w_{i}\right]$ of these vertices, and removing these vertices from $G_{r}$ we obtain a locally $P_{5}$ graph $G_{r}^{\prime}$ of order

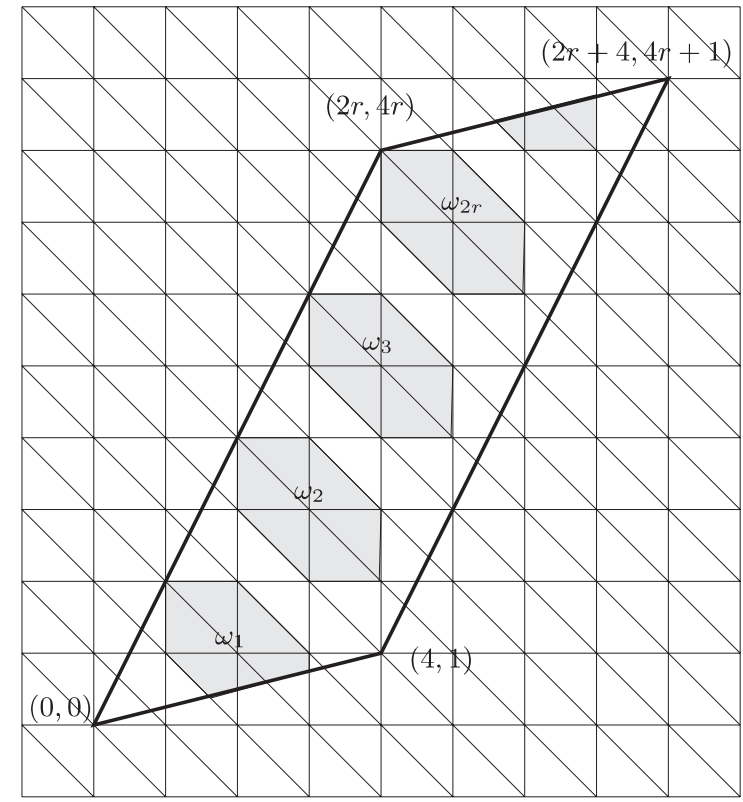

Figure 2: The parallelogram $\mathcal{P}_{r}$, for $r=2$.

12r. Let us call $\mathcal{S}_{r}$ the surface triangulated by $G_{r}^{\prime}$, which is a torus with $2 r$ open disks removed. All the vertices of $G_{r}^{\prime}$ lie in the border of $\mathcal{S}_{r}$. The connected components of the border of $\mathcal{S}_{r}$ are the hexagons $H_{1}, H_{2}, \ldots, H_{2 r}$ which were the open neighbourhoods of the removed vertices $w_{1}, w_{2}, \ldots, w_{2 r}$ of $G_{r}$.

Consider the locally $P_{4}$ graph $C_{12}^{2}$ in figure 3 . This graph gives us a Whitney triangulation of a cylinder, all the vertices lie in the border whose connected components $B_{1}$ and $B_{2}$ are induced hexagons of $C_{12}^{2}$.

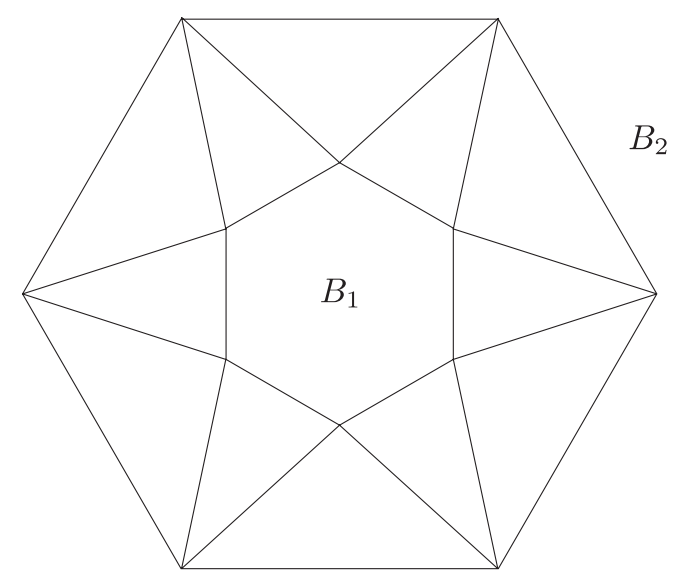

Figure 3: The graph $C_{12}^{2}$ 
Now, take the surface $\mathcal{S}_{r}$ (with its Whitney triangulation given by the graph $G_{r}^{\prime}$ ) and $r$ different copies of the cylinder (with the Whitney triangulation given by $C_{12}^{2}$ ). For the first copy, identify $B_{1}$ with $H_{1}$ and $B_{2}$ with $H_{r+1}$ in an orientable manner, so a handle is glued to $\mathcal{S}_{r}$. For the second copy, identify $B_{1}$ with $H_{2}$ and $B_{2}$ with $H_{r+2}$, so a second handle is glued to $\mathcal{S}_{r}$. Continuing in this way, we obtain at the end a closed surface $\mathcal{S}_{r}^{\prime}$ which is a sphere with $r+1$ handles. The graph $\bar{G}_{r}$ obtained from $G_{r}^{\prime}$ and the $r$ copies of $C_{12}^{2}$ by the above method has $12 r$ vertices and is the 1skeleton of a triangulation of our surface $\mathcal{S}_{r}^{\prime}$.

As we want $\bar{G}_{r}$ to be locally $C_{7}$ we have to take care so that the triangles in $\bar{G}_{r}$ are exactly the triangles already present $\left(16 r\right.$ in $G_{r}^{\prime}$ and 12 in each copy of $C_{12}^{2}$ ). This fails when two vertices $x \in H_{i}$ and $y \in H_{i+r}$ with $d(x, y)<3$ in $G_{r}^{\prime}$ are identified with adjacent vertices in the $i$-th copy of $C_{12}^{2}$. Since $d\left(H_{i}, H_{r+i}\right)=r$, there is no problem for $r \geq 3$.

In case $r=2$, there is an essentially unique way to glue the 2 copies of $C_{12}^{2}$ in such a way that no new triangles are created, and this produces a triangulation of the orientable closed surface of genus 3 (the "triple torus"). We verified this by computer using GAP [4]. It can be shown that the double torus does not admit a locally $C_{7}$ triangulation.

Notice that for $r \geq 3$ the construction allows more freedom at the time of gluing (so in principle more than one example may have been constructed at each genus $g>3$ ) and that even nonorientable surfaces are obtained gluing one handle in a non-orientable manner. So we have proved:

Theorem 3.1 Every orientable surface of genus at least 3 , and every non-orientable surface with even Euler characteristic $\chi \leq-6$ admits a locally $C_{7}$ triangulation $G$. For any such $G, K(G)$ is a positive answer to Prisner's open problem 1 in [11].

\section{References}

[1] Claude Berge. Hypergraphs. North-Holland Publishing Co., Amsterdam, 1989. Combinatorics of finite sets, Translated from the French.

[2] Morton Brown and Robert Connelly. On graphs with a constant link. In New directions in the theory of graphs (Proc. Third
Ann Arbor Conf., Univ. Michigan, Ann Arbor, Mich., 1971), pages 19-51. Academic Press, New York, 1973.

[3] C. H. Dowker. Homology groups of relations. Ann. of Math. (2), 56:84-95, 1952.

[4] The GAP Group, Aachen, St Andrews. GAP - Groups, Algorithms, and Programming, Version 4.2, 2000. (http://www-gap-system.org).

[5] Nora Hartsfield and Gerhard Ringel. Clean triangulations. Combinatorica, 11(2):145$155,1991$.

[6] P. J. Hilton and S. Wylie. Homology theory: An introduction to algebraic topology. Cambridge University Press, New York, 1960.

[7] F. Larrión and V. Neumann-Lara. Locally $C_{6}$ graphs are clique divergent. Discrete Math., 215(1-3):159-170, 2000.

[8] F. Larrión, V. Neumann-Lara, and M. A. Pizaña. Whitney triangulations, local girth and iterated clique graphs. Discrete Math., 258(1-3):123-135, 2002.

[9] Erich Prisner. Homology of the line graph and of related graph-valued functions. Arch. Math., 56(4):400-404, 1991.

[10] Erich Prisner. Convergence of iterated clique graphs. Discrete Math., 103(2):199-207, 1992.

[11] Erich Prisner. Graph dynamics. Longman, Harlow, 1995.

[12] C. P. Rourke and B. J. Sanderson. Introduction to piecewise-linear topology. SpringerVerlag, Berlin, 1982. Reprint.

[13] Edwin H. Spanier. Algebraic topology. Springer-Verlag, New York, 1981. Corrected reprint.

[14] Jayme L. Szwarcfiter. A survey on clique graphs. In Recent Advances in Algorithms and Combinatorics. C. Linhares and B. Reed, eds., Springer-Verlag. To appear.

[15] Jayme L. Szwarcfiter. Recognizing cliqueHelly graphs. Ars Combin., 45:29-32, 1997.

[16] W. T. Tutte. A census of plane triangulations. Canad. J. Math., (14):21-28, 1962.

[17] H. Whitney. A theorem on graphs. Ann. Math., 32(2):378-390, 1931. 